Revista Verde de Agroecologia e Desenvolvimento Sustentável

http://www.gvaa.com.br/revista/index.php/RVADS

ARTIGO CIENTÍFICO

\title{
Efeito do nitrogênio e do lodo de esgoto na nutrição do feijoeiro
}

\section{Effect of nitrogen and sewage sludge on bean plant nutrition}

\author{
Thomaz F. Lobo ${ }^{1}$, Helio G. Filho ${ }^{2}$, Leonardo T. Büll ${ }^{3}$, Flavia L. P. de Souza ${ }^{4}$
}

\begin{abstract}
Resumo: No Brasil, a disposição final do lodo geralmente é o aterro sanitário, sendo que desta forma apresenta elevados custos e agrava ainda mais o problema com o manejo do lixo urbano uma das maneiras eficazes do aproveitamento é na agricultura. O objetivo deste trabalho foi verificar o efeito do $\mathrm{N}$ e do lodo de esgoto na nutrição do feijoeiro. O experimento foi conduzido na Fazenda Experimental São Manuel, pertencente à Faculdade de Ciências Agronômicas da UNESP de Botucatu. Foi adotado o delineamento experimental em blocos casualizados, constituídos por 6 tratamentos e 4 repetições assim definidos: T0 - sem adubação nitrogenada; T1 - adubação química nitrogenada de acordo com a necessidade da cultura para o estado de São Paulo; T2 - 50\% adubação nitrogenada proveniente do lodo de esgoto e 50\% na forma mineral; T3 - $100 \%$ adubação nitrogenada proveniente do lodo de esgoto; T4 - 150\% adubação nitrogenada proveniente do lodo de esgoto; T5 $200 \%$ adubação nitrogenada proveniente do lodo de esgoto. Os parâmetros avaliados foram os teores foliares e a exportação de nutrientes. O aumento da dose de lodo influenciou nos teores foliares de Ca, P, Mg, Cu, Fe e Zn. A exportação de N, P, K, Ca, $\mathrm{Mg}, \mathrm{B}, \mathrm{Cu}, \mathrm{Fe}, \mathrm{Mn}$ e $\mathrm{Zn}$ influenciaram com o aumento da dose de lodo. $\mathrm{O} \mathrm{N}$ não interferiu nos teores foliares. $\mathrm{O} \mathrm{N}$ proporcionou uma maior exportação de $\mathrm{Cu}$ e $\mathrm{Mn}$.
\end{abstract}

Palavras chaves: exportação de nutrientes, sustentabilidade, materia organica, compostagem, diagnose foliar

Abstract: In Brazil, the final disposal of sludge is generally on a landfill site, requiring high costs and worsening the problem of urban waste management, which has as one of the most effective solutions the use in agriculture. The aim of this work was to verify the effect of $\mathrm{N}$ and sewage sludge on bean plant nutrition. The experiment was carried out in São Manuel Experimental Farm, which belongs to Faculdade de Ciências Agronômicas - UNESP, Botucatu. The adopted experimental design was in randomized blocks, with 6 treatments and 4 replicates, defined as follows: T0 - without nitrogen fertilization; T1 - nitrogen chemical fertilization according to the needs of the culture for São Paulo State; T2 - 50\% nitrogen fertilization from sewage sludge and 50\% in the mineral form; T3 - 100\% nitrogen fertilization from sewage sludge; T4 - 150\% nitrogen fertilization from sewage sludge; T5 - 200\% nitrogen fertilization from sewage sludge. The evaluated parameters were leaf content and nutrient exportation. The increase in sludge levels influenced the leaf content of $\mathrm{Ca}, \mathrm{P}, \mathrm{Mg}, \mathrm{Cu}, \mathrm{Fe}$ and $\mathrm{Zn}$. The exportation of $\mathrm{N}, \mathrm{P}, \mathrm{K}, \mathrm{Ca}, \mathrm{Mg}, \mathrm{B}, \mathrm{Cu}, \mathrm{Fe}, \mathrm{Mn}$ and $\mathrm{Zn}$ was also influenced by the increase in sludge levels. $\mathrm{N}$ did not interfere with leaf content and led to an increase in $\mathrm{Cu}$ and $\mathrm{Mn}$ exportation.

Keywords: nutrient exportation, sustainability, organic matter, compost, leaf analysis

\footnotetext{
*Autor para correspondência

Recebido para publicação em 25/04/2015; aprovado em 28/05/2015

${ }^{1}$ Eng. Agr. Dr. Prof. - USC - Universidade do Sagrado Coração, Bauru - SP. E-mail: thomaz.lobo@ superig.com.br

${ }^{2}$ Eng. Agr. Dr. Prof. - USC - Universidade do Sagrado Coração, Bauru - SP - UNESP - Departamento de Recursos Naturais/Ciência do Solo e Nutrição Mineral de Plantas. E-mail: heliograsi@fca.unesp.br

${ }^{3}$ Eng. Agr. Dr. Prof. - USC - Universidade do Sagrado Coração, Bauru - SP - UNESP - Departamento de Recursos Naturais/Fertilidade do Solo. E-mail: bull@fca.unesp.br

${ }^{4}$ Estudante de Eng. Agr. - USC - Universidade do Sagrado Coração, Bauru-SP. E-mail: flavialuizesouza@ hotmail.com
} 


\section{INTRODUÇÃO}

Segundo Valderrama et al. (2009) o feijão além da grande importância econômica, é muito usado pelos brasileiros, por conseguinte é uma das fundamentais culturas no Brasil. O excesso, a falta e a eficiência dos fertilizantes são fatores que ocasionam a diminuição da produtividade., devido o feijoeiro ser muito exigente em nutrientes, principalmente o $\mathrm{N}$ e P. O uso de fontes nitrogenadas e fosfatadas de liberação gradual pode trazer diminuição no custo de produção e menores impactos ambientais, reduzindo as perdas por fixação $\mathrm{P}$, volatização e lixiviação $\mathrm{N}$

Cada vez mais a sociedade solicita o controle e o avanço das situações ambientais para as autoridades e empresas públicas e privadas. $\mathrm{O}$ esgoto urbano está entre os maiores poluidores dos mananciais, porém fornece um lodo rico em matéria orgânica e nutrientes, que é descartado e considerado um problema pelas Estações de Tratamento de Esgoto (ETEs). Algumas das alternativas muito proveitosas para a área agrícola são: recuperação de solos; fertilizantes e compostagem. Após, o lodo de esgoto ser tratado apresenta propriedades que possibilita sua utilização agrícola de maneira coerente e ambientalmente segura (BARBOSA; FILHO, 2006).

No Brasil, a disposição final do lodo geralmente é o aterro sanitário. Alem do alto custo, que pode chegar a $50 \%$ do custo operacional de uma ETE, a disposição de um resíduo com elevada carga orgânica no aterro, agrava ainda mais o problema com o manejo do lixo urbano (BETIOL \& CAMARGO, 2007).

Segundo Betil \& Camargo (2007), em países da Europa e América do norte, o lodo geralmente é incinerado, depositado em aterros sanitários ou utilizados em áreas agrícolas, dependendo das características do resíduo.

$\mathrm{Na}$ maioria dos países existem normas que regulamentam o destino do lodo, garantindo uma disposição segura. A adição ao solo parece ser a melhor opção sob o ponto de vista econômico e ambiental, uma vez que apresenta o menor custo e promove a reciclagem de matéria orgânicas e nutrientes (BETIOL \& CAMARGO, 2007).

O uso agrícola do lodo de esgoto como adubo orgânico é considerado hoje como alternativa promissora de disposição final deste resíduo, devido a sua sustentabilidade, ou seja, com a sua utilização pode-se diminuir a adubação mineral e fornecer uma fonte orgânica e seu efeito pode ser potencializado, aliando-se a utilização agrícola e a recuperação de áreas degradadas (KITAMURA et al., 2008).

Lobo et al. (2012) verificaram que o aumento das doses de lodo de esgoto no solo promoveu uma absorção significativa de $\mathrm{N}, \mathrm{P}, \mathrm{Ca}$ e Mg em plantas de sorgo granífero. Entretanto, Wisniewski et al. (1996) estudando a viabilidade do uso do lodo de esgoto na recuperação de áreas degradadas pela mineração de calcário, relataram que o lodo parece ser mais indicado para melhorar as condições físicas do solo do que para fornecimento de nutrientes para a planta.

A utilização de lodo de esgoto é uma prática altamente promissora para o desenvolvimento de sistemas agrícolas sustentáveis. No entanto, normas rígidas pela elaboração de projetos devem ser seguidas para minimizar o impacto desta prática no ambiente, a norma técnica P4.240 (CONAMA, 2006).
Cunninchan et al. (1975) incorporando ao solo lodo de esgoto sem tratamento prévio, obtiveram aumento na produção de grãos de milho, que relacionou maior disponibilidade de $\mathrm{N}, \mathrm{P}$ e K.

A utilização do lodo de esgoto em solos agrícolas tem como principal beneficio, a incorporação dos macronutrientes ( $\mathrm{N}$ e $\mathrm{P}$ ) e dos micronutrientes ( $\mathrm{Zn}, \mathrm{Cu}, \mathrm{Fe}, \mathrm{Mn}$ e $\mathrm{Mo}$ ) (BETIOL \& CAMARGO, 2007).

No experimento de produção de milho safrinha realizado por Barbosa et al (2007) constatou-se resultados de acordo com o uso do lodo de esgoto calado. Os nutrientes disponibilizados no solo pelo resíduo possibilitou o aumento da produtividade do milho, portanto a reciclagem agrícola do Iodo de esgoto é uma das maneiras mais favorável em termos técnicos, econômicos e ambientais.

O aumento da dose de lodo de esgoto promoveu incremento no número de vagens, rendimento de matéria seca, rendimento de grãos e na massa de 1000 grãos. O N promoveu o aumento do rendimento de grãos e da massa de matéria seca do feijoeiro (LOBO et al., 2012).

Pode-se dizer que, normalmente, o lodo de esgoto é uma fonte de nutrientes para as culturas. No entanto, é preciso conhecimento da sua composição, para se calcular a quantidade adequada a ser incorporada, sem correr o risco de toxicidade as plantas e em certas situações aos animais e ao homem, como também não poluir o ambiente (CONAMA,2006).

Os lodos de esgotos são, de uma maneira geral, fertilizantes nitrogenados. Além do N, os lodos de esgotos contém $\mathrm{P}$ e micronutrientes essenciais, como $\mathrm{Fe}$ e $\mathrm{Zn}$. Normalmente a concentração de K no lodo de esgoto é baixa para suprir as necessidades das culturas agrícolas. Quando aplicado em taxas agronômicas, os lodos de esgotos podem reduzir em muito o custo de produção, pela menor utilização de fertilizantes minerais solúveis (VALDERRAMA et al., 2009).

Corrêa et al (2008) e Corrêa et al (2007) utilizaram do lodo de esgoto calado no solo, por conseguinte ocorreu o aumento de pH. Segundo Campanharo et al (2010) a acidez do solo $\left(\mathrm{Al}^{3+} \mathrm{e} \mathrm{H}^{+}+\mathrm{Al}^{3+}\right)$ acarretam consequências negativas no desenvolvimento e na nodulação do feijoeiro.

$\mathrm{O}$ feijoeiro requer quantidades altas de $\mathrm{N}$ e $\mathrm{K}$ e quantidades relativamente baixas de $\mathrm{P}, \mathrm{Ca}, \mathrm{Mg}$ e $\mathrm{S}$. Estes nutrientes são obtidos dos solos e dos fertilizantes aplicados com exceção do $\mathrm{N}$, que pode ser fornecido pela fixação biológica de $\mathrm{N}$ pela simbiose com bactérias do gênero Rhizobium (EMBRAPA, 1993).

Existe um descrédito quanto a capacidade do feijoeiro fixar $\mathrm{N}$ atmosférico em quantidades suficientes para suprir a exigência da produção. Em associação com Rhizobium, é recomendado indistintamente o uso de $\mathrm{N}$ para a cultura (EMBRAPA, 1993). Entretanto, resultados de vários anos de pesquisa apontam na direção contraria, sugerindo que é possível que a cultura do feijoeiro se beneficie, em nível de campo, da fixação do N (OLIVEIRA; ARAUJO; DUTRA 1996).

$\mathrm{O}$ feijoeiro irrigado pode responder a dose de $\mathrm{N}$ em cobertura acima de $150 \mathrm{~kg} \mathrm{ha}^{-1}$ e que é necessário parcelar a dose de duas a três vezes durante o ciclo. (BARBOSA; FILHO, 2006).

Lobo et al., (2012) demonstraram que a aplicação de fertilizantes nitrogenadas no feijoeiro pode ser substituída por 
quantidades adequadas de lodo de esgoto e ficou também evidenciado que a aplicação de lodo pode estimular a nodulação e a fixação simbiótica de $\mathrm{N}_{2}$ pelas estirpes nativas de rizobio no feijoeiro.

O objetivo deste trabalho foi verificar o efeito do $\mathrm{N}$ e do lodo de esgoto na nutrição e na exportação pelo grão no feijoeiro.

\section{MATERIAL E MÉTODOS}

O experimento foi conduzido na Fazenda Experimental São Manuel, pertencente à Faculdade de Ciências Agronômicas da UNESP de Botucatu, localizada no município de São Manuel a $22^{\circ} 25^{\prime}$ Latitude Sul, $48^{\circ} 34^{\prime}$ ' Longitude Oeste, com altitude de 750 metros.

Antecedendo a instalação do experimento, foram cultivados 2 ciclos consecutivos de girassol semeados em dezembro de 2004 e novembro de 2005, respectivamente, com aplicações de lodo de esgoto e cultivado com trigo e triticale, avaliando o efeito residual dessas duas aplicações de lodo de esgoto, em abril de 2006.

Foi cultivado em março de 2007 a cultura da aveia com aplicação de lodo de esgoto com intuito de fornecer palha para entrar no sistema plantio direto. Em dezembro de 2007 foi aplicado o lodo de esgoto compostado em sistema de semeadura direta para o cultivo do feijão comum.

Foi adotado o delineamento experimental em blocos casualizados, constituídos por 6 tratamentos e 4 repetições assim definidos (PIMENTEL, 2000): T0 - sem adubação nitrogenada; T1 - adubação química nitrogenada de acordo com Raij et al., (1997); T2 - 50\% adubação nitrogenada proveniente do lodo de esgoto e $50 \%$ na forma mineral; T3 $100 \%$ adubação nitrogenada proveniente do lodo de esgoto; T4 - 150\% adubação nitrogenada proveniente do lodo de esgoto; T5 - 200\% adubação nitrogenada proveniente do lodo de esgoto.

Cada parcela apresentou uma área de 100 metros quadrados $(14 \times 7,2 \mathrm{~m})$ com um espaço de $3 \mathrm{~m}$ entre parcelas do mesmo bloco. O espaço de um bloco a outro foi de $1,8 \mathrm{~m}$.

Neste ensaio foi utilizado um lodo de esgoto compostado com bagaço de cana, tendo como a principal finalidade a eliminação de patógenos. No processo de compostagem a temperatura chega a, aproximadamente, $70^{\circ} \mathrm{C}$, o que elimina grande parte dos patógenos existentes no resíduo.

O composto apresenta as características descritas na Tabela 1 (LANARV, 1988).

Tabela 1. Características químicas do composto de lodo de esgoto

\begin{tabular}{|c|c|c|c|c|c|c|c|c|c|c|c|c|c|c|c|}
\hline $\mathrm{N}$ & $\mathrm{P}_{2} \mathrm{O}_{5}$ & $\mathrm{~K}_{2} \mathrm{O}$ & Umid. & $\mathrm{MO}$ & $\mathrm{C}$ & $\mathrm{Ca}$ & $\mathrm{Mg}$ & $\mathrm{S}$ & $\mathrm{Na}$ & $\mathrm{Cu}$ & $\mathrm{Fe}$ & $\mathrm{Mn}$ & $\mathrm{Zn}$ & $\mathrm{C} / \mathrm{N}$ & $\mathrm{pH}$ \\
\hline-- & ------. & ---- & $\%$ & --- & --- & & & & ----- & --.--. & $\mathrm{ng} \mathrm{kg}^{-1}$ & 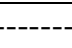 & & & \\
\hline 2,4 & 2,0 & 0,4 & 50 & 55,0 & 31,0 & 1,9 & 0,3 & 1,6 & 1340 & 304 & 23250 & 472 & 3750 & $13 / 1$ & 6,6 \\
\hline
\end{tabular}

Adotou-se o seguinte cálculo para determinação da dose de lodo de esgoto utilizada nos tratamentos: Cada $100 \mathrm{~kg}$ de lodo de esgoto na base seca contém $2,4 \mathrm{~kg}$ de $\mathrm{N}$; Considerando o teor de água de 50 , ou seja, $50 \%$ base seca, teremos $1,2 \%$ de $\mathrm{N}$ na base úmida; Considerando que $30 \%$ do $\mathrm{N}$ serão mineralizados de lodo de esgoto (CONAMA, 2006), teremos 0,36 \% de $\mathrm{N}$ mineralizado; As quantidades de lodo de esgoto destinadas para cada tratamento foram: T2 $10.000 \mathrm{~kg} \mathrm{ha}^{-1} ; \mathrm{T} 3-20.000 \mathrm{~kg} \mathrm{ha}^{-1} ; \mathrm{T} 4-30.000 \mathrm{~kg} \mathrm{ha}^{-1} ; \mathrm{T} 5-$ $40.000 \mathrm{~kg} \mathrm{ha}^{-1}$.
Foi executada uma calagem nas parcelas em que houve necessidade, utilizando uma dosagem de 1,4 ton $\mathrm{ha}^{-1}$ de calcário dolomítico com PRNT de $90 \%$ e teor de $\mathrm{CaO}$ de $32 \%$ e $\mathrm{MgO}$ de $13 \%$. Essa dosagem foi baseada nas análises de solo retiradas no final do ensaio anterior.

O cálculo dessa dosagem foi fundamentado na parcela que apresentou uma V\% menor, que foi a parcela que aplicou uma maior dose de lodo de esgoto, conforme a Tabela 2. Nas parcelas que apresentaram uma $\mathrm{V} \%$ superior a $70 \%$ não foram realizadas a calagem.

Tabela 2- Características químicas do solo da parcela de menor V\% (básicas)

\begin{tabular}{|c|c|c|c|c|c|c|c|c|c|c|c|}
\hline Prof. & $\mathrm{pH}$ & M.O. & $\mathrm{P}($ res. $)$ & $\mathrm{H}+\mathrm{Al}$ & $\mathrm{Al}^{+3}$ & $\mathrm{~K}^{+}$ & $\mathrm{Ca}^{+2}$ & $\mathrm{Mg}^{+2}$ & SB & $\mathrm{T}$ & $\mathrm{V}$ \\
\hline $\mathrm{Cm}$ & $\mathrm{CaCl}_{2}$ & $\mathrm{~g} \mathrm{dm}_{3}^{-}$ & $\mathrm{mg} \mathrm{dm} \mathrm{m}^{-3}$ & & --- & --- & - & $\mathrm{l}_{\mathrm{c}} \mathrm{d}$ & & & $\%$ \\
\hline $0-20$ & 4,7 & 30 & 23 & 28 & - & 2,2 & 17 & 5 & 24 & 53 & 46 \\
\hline
\end{tabular}

Após 6 dias da aplicação de calcário, foi efetuada a aplicação do composto de lodo de esgoto e a aplicação de gliphosate.

A semeadura foi realizada 5 dias após a aplicação de Gliphosate. Efetuou-se o tratamento de sementes com Carboxin para o controle preventivo de moléstias. A quantidade foi de 13 sementes em 1 metro, em espaçamento entre linha de 0,45 metros, totalizando 288.888 sementes em 1 ha. A profundidade de semeadura foi de $3 \mathrm{~cm}$ aproximadamente.

A adubação de semeadura foi efetuada com base na média da análise de solo da cultura anterior, utilizando como fonte de $\mathrm{N}$ a uréia de $\mathrm{P}$ superfosfato triplo e $\mathrm{K}$ cloreto de potássio. Foi efetua a adubação nitrogenada no tratamento $\mathrm{T} 1$ na dosagem de $10 \mathrm{~kg} \mathrm{ha}^{-1}$ de $\mathrm{N}$ utilizando como fonte a uréia.
Aos 11 dias após a semeadura, ocorreu incidência de plantas de Avena strigosa, aveia preta, as quais foram controladas com o Fluazifop- $\mathrm{p}$ - butil, na dosagem de 1,6 L $\mathrm{ha}^{-1}$.

Foi realizada uma adubação nitrogenada de cobertura, aos 29 dias após a semeadura, nos tratamentos T1 e T2 nas dosagens de 60 e $35 \mathrm{~kg} \mathrm{ha}^{-1}$ de $\mathrm{N}$, respectivamente. A fonte utilizada foi a uréia.

Aos 36 dias após a semeadura, foi constatada na área a incidência de Raphanus raphanistrum (nabiça). Aplicou-se o Bentazone, na dosagem de $0,8 \mathrm{~L} \mathrm{ha}^{-1}$, em um volume de calda de $250 \mathrm{~L} \mathrm{ha}^{-1}$.

Aos 44 dias após a semeadura, foi aplicado o Chlorpyrikos, devido à incidência de Pseudoplusia includens (lagarta). A dosagem utilizada foi de $0,5 \mathrm{~L} \mathrm{ha}^{-1} \mathrm{em} \mathrm{um}$ volume de calda de $200 \mathrm{~L} \mathrm{ha}^{-1}$. 
Aos 52 dias após a semeadura, foi empregado o Tebucanazole para o controle de Uromyces phaseoli (ferrugem). Foi utilizada uma dosagem de $0,3 \mathrm{~L} \mathrm{ha}^{-1}$, em um volume de calda de $200 \mathrm{~L} \mathrm{ha}^{-1}$.

Aos 66 dias após a semeadura, foi utilizado o Metamidophós para o controle de Deois flavopicta (cigarrinha das pastagens). A dosagem utilizada foi de $0,3 \mathrm{~L} \mathrm{ha}^{-1}$ e o volume de calda de $200 \mathrm{~L} \mathrm{ha}^{-1}$.

A colheita foi realizada 95 dias após a semeadura.Por ocasião do início da floração, foram coletadas 10 amostras de folhas do feijoeiro de cada parcela, a primeira folha amadurecida a partir da ponta do ramo (MALAVOLTA; VITTI; OLIVEIRA, 1997).

Posteriormente levadas à estufa, com circulação de ar forçada até atingir um peso constante. Em seguida foram moídas e analisadas no Laboratório de Nutrição Mineral de
Plantas, do Departamento de Recursos Naturais/ Ciência do Solo/ Botucatu/ SP, determinando os teores de N, P, K, Ca, $\mathrm{Mg}, \mathrm{S}, \mathrm{B}, \mathrm{Cu}, \mathrm{Fe}, \mathrm{Mn}$ e $\mathrm{Zn}$ (MALAVOLTA; VITTI; OLIVEIRA, 1997).

A determinação do teor de nutrientes no grão foi utilizada da mesma maneira descrita para o teor nas folhas.Após a determinação dos teores, foi calculada, considerando a massa dos grãos de uma planta, o acúmulo de nutrientes nos grãos e, conseqüentemente, a exportação de nutrientes.

Os dados climatológicos utilizados no presente estudo foram apenas os ligados à variável precipitação das chuvas $(\mathrm{mm})$ e temperatura. Foi instalado no local do ensaio um pluviômetro para medir a precipitação. Durante o ciclo da cultura houve uma precipitação total de $733 \mathrm{~mm}$, como se observa na Figura 1.
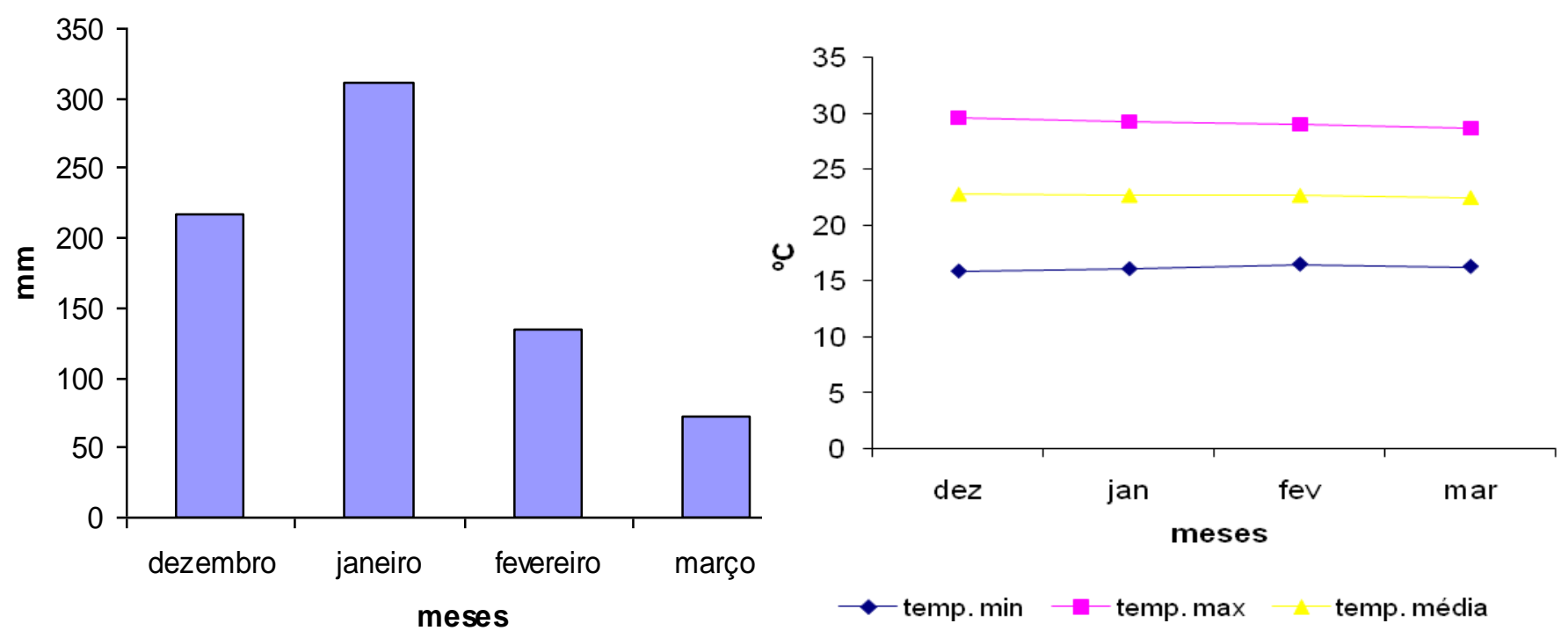

Figura 1. Precipitação Pluviométrica, temperatura mínima média, média e máxima média no ciclo do feijoeiro de dezembro a março no ano $2007 / 2008$

Os dados de temperatura mínima, média e máxima média foram fornecidos pela estação climatológica da fazenda experimental de São Manuel pertencente ao Departamento Recursos Naturais, Área de Ciências Ambientais da Faculdade de Ciências Agronômicas de Botucatu, obtidos na própria Fazenda Experimental São Manuel, onde existe uma estação climatológica.

Os dados foram submetidos a análise de variância e as média comparadas pelo teste de Duncan 5\%, para todos os tratamentos, realizando o programa de estatística Saeg, (1993). Nos tratamentos T0, T3, T4 e T5, que sofreram o aumento da dose de lodo, foi efetuada uma regressão para os parâmetros que houveram diferença estatística com o aumento da dose de lodo compostado.

\section{RESULTADOS E DISCUSSÃO}

Segundo Malavolta et al. (1997), os teores foliares de macronutrientes adequados para o feijoeiro são os seguintes: $\mathrm{N}\left(30-50 \mathrm{~g} \mathrm{~kg}^{-1}\right), \mathrm{P}\left(2-3 \mathrm{~g} \mathrm{~kg}^{-1}\right), \mathrm{K}\left(20-25 \mathrm{~g} \mathrm{~kg}^{-1}\right), \mathrm{Ca}(15-20 \mathrm{~g}$ $\left.\mathrm{kg}^{-1}\right), \mathrm{Mg}\left(4-7 \mathrm{~g} \mathrm{~kg}^{-1}\right)$ e S $\left(5-10 \mathrm{~g} \mathrm{~kg}^{-1}\right)$.

Verifica-se na Tabela 3 que para os teores de $\mathrm{N}$ os tratamentos T3, T4 e T5 estão acima dos valores adequados e os tratamentos (T0, T1 e T2) estão adequados. Entre os tratamentos não ocorreu variação. Arf et al. (2008), não observaram diferença nos teores de $\mathrm{N}$ nas folhas de feijão utilizando as seguintes doses de $\mathrm{N}(0,25,50,75,100$ e 125 $\mathrm{kg} \mathrm{ha}^{-1}$ ). Binotti et al. (2007), verificaram que os tratamentos onde não foi aplicado $\mathrm{N}$ e onde foi aplicado $75 \mathrm{~kg} \mathrm{ha}^{-1}$ nos estágios V3 e V4, ocorreu uma diferença significativa nos teores foliares, no primeiro cultivo de feijão após milho.

Quanto ao P observa-se que todos os tratamentos apresentaram valores acima dos níveis adequados. Entre os tratamentos o T5 apresentou superior aos tratamentos (T2 e T4) como pode ser observado na Tabela 3, isto pode ser explicado que o lodo apresenta um teor elevado de $\mathrm{P}$ como consta na Tabela 1.

Para o $\mathrm{K}$ os tratamentos que apresentaram superiores ao nível adequado foram o T0, T2, T3, T4 e T5, somente o T1 apresentou teor adequado (Tabela 3 ).

No $\mathrm{Ca}$ todos os tratamentos apresentaram teores abaixo do adequado (15-20 $\mathrm{g} \mathrm{kg}^{-1}$ ), acredita-se que esta relacionada aos altos teores apresentados de $\mathrm{N}\left(\mathrm{NH}_{4}^{+}\right)$e $\mathrm{K}^{+}$que pode ter inibido a absorção do $\mathrm{Ca}$, porém não foi encontrada deficiência visual de $\mathrm{Ca}$ nas folhas.

$\mathrm{O}$ tratamento T5 apresentou-se um teor de Ca superior que o T3 (Tabela 3). Na Figura 2 observa-se que apresenta uma 
queda no teor de Ca e em seguida um aumento em função do aumento da dose de lodo compostado.

$\mathrm{O} \mathrm{Mg}$ todos apresentara teores abaixo do adequado, isto pode ter ocorrido devido aos teores elevados de $\mathrm{K}$ e ou $\mathrm{N}$ inibiu o $\mathrm{Mg}$, porem não foi apresentado visualmente sintomas de deficiência. Em função do aumento da dose de lodo o $\mathrm{Mg}$ se comportou da mesma forma que o Ca (Figura 2).

Tabela. 3 Teores foliares de macronutrientes no feijão

\begin{tabular}{lllllll}
\hline Trat & $\mathrm{N}$ & $\mathrm{P}$ & $\mathrm{K}$ & $\begin{array}{c}\mathrm{Ca} \\
\mathrm{g} \mathrm{kg}^{-1}\end{array}$ & $\mathrm{Mg}$ & $\mathrm{S}$ \\
\hline T0 & $48,4 \mathrm{ab}$ & $6,3 \mathrm{ab}$ & $25,2 \mathrm{ab}$ & $7,0 \mathrm{ab}$ & $3,6 \mathrm{ab}$ & $2,7 \mathrm{a}$ \\
T1 & $47,0 \mathrm{~b}$ & $6,0 \mathrm{ab}$ & $24,0 \mathrm{~b}$ & $6,4 \mathrm{ab}$ & $3,7 \mathrm{ab}$ & $2,3 \mathrm{~b}$ \\
T2 & $47,8 \mathrm{ab}$ & $5,6 \mathrm{~b}$ & $25,4 \mathrm{ab}$ & $7,2 \mathrm{ab}$ & $3,6 \mathrm{ab}$ & $2,4 \mathrm{ab}$ \\
T3 & $52,0 \mathrm{a}$ & $6,2 \mathrm{ab}$ & $25,2 \mathrm{ab}$ & $5,8 \mathrm{~b}$ & $3,2 \mathrm{ab}$ & $2,8 \mathrm{a}$ \\
T4 & $52,0 \mathrm{a}$ & $5,7 \mathrm{~b}$ & $25,2 \mathrm{ab}$ & $6,8 \mathrm{ab}$ & $3,1 \mathrm{~b}$ & $2,6 \mathrm{ab}$ \\
T5 & $51,2 \mathrm{ab}$ & $6,9 \mathrm{a}$ & $29,2 \mathrm{a}$ & $9,8 \mathrm{a}$ & $3,8 \mathrm{a}$ & $2,7 \mathrm{a}$ \\
\hline F & 2,55 & 1,76 & 1,29 & 2,01 & 2,01 & 2,44 \\
Média & 49,7 & 6,1 & 25,7 & 7,2 & 3,5 & 2,6 \\
CV & 6,34 & 13,13 & 13,68 & 30,42 & 13,59 & 10,65 \\
\hline
\end{tabular}

Médias seguidas pelas mesmas letras não diferem entre si pelo teste de Duncan 5\% de probabilidade. T0 - sem N, T1 -100\% AM, T2 - 50\% AM + 50\% LE, T3 - 100\% LE, T4 - 150\% LE, T5 - 200\% LE. AM - Adubação mineral; LE - Lodo de esgoto
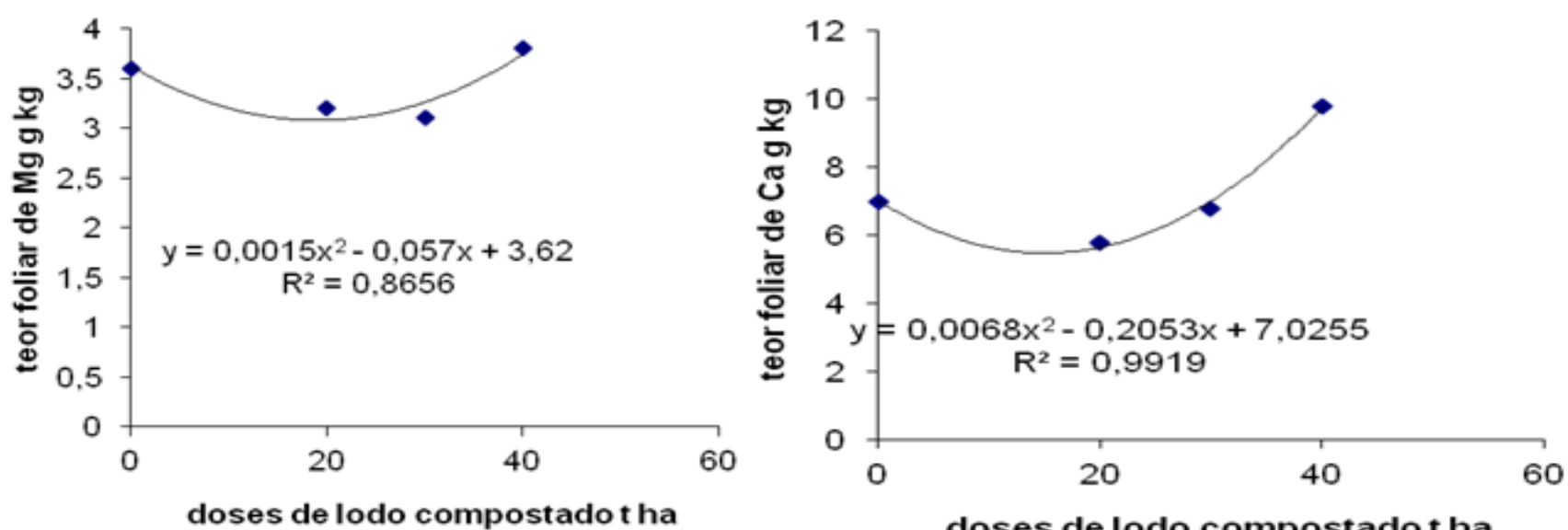

Figura 2 Teores foliares de macronutrientes no feijão em função da dose de lodo compostado

O S apresentou teores abaixo do adequado para todos os tratamentos, como em todas as culturas utilizadas no sistema utilizou-se como fonte de $\mathrm{K}$ o $\mathrm{KCl}$, o $\mathrm{Cl}$ pode ter inibido a absorção pelo S. Observa-se na Tabela 3 que os tratamentos T0, T3 e T5 apresentaram superiores ao T1 e os tratamentos T2 e T4 não diferiram estatisticamente com os outros tratamentos.

José (2001) constatou que o aumento das doses de $\mathrm{N}$ apresentou um decréscimo dos teores de $\mathrm{Mg}$ e $\mathrm{S}$ em feijão, pode-se observar que houve um decréscimo também dos teores foliares de $\mathrm{S}$ onde foi aplicado o $\mathrm{N}$ e onde não aplicou N.

Para os micronutrientes os teores considerados adequados, segundo (Malavolta et al. 1997), são os seguintes: B (30 - 60 $\left.\mathrm{mg} \mathrm{kg}{ }^{-1}\right), \mathrm{Cu}\left(10-20 \mathrm{mg} \mathrm{kg}^{-1}\right)$, Fe $\left(100-450 \mathrm{mg} \mathrm{kg}^{-1}\right), \mathrm{Mn}$ $\left(30-300 \mathrm{mg} \mathrm{kg}^{-1}\right)$ e $\mathrm{Zn}\left(20-100 \mathrm{mg} \mathrm{kg}^{-1}\right)$.

Pode-se observar na Tabela 4 que todos os micronutrientes apresentaram teores adequados.

Tabela 4 Teores foliares de micronutrientes no feijão

\begin{tabular}{cccccc}
\hline Tratamento & B & $\mathrm{Cu}$ & $\begin{array}{c}\text { Fe } \\
\mathrm{mg} \mathrm{kg}^{-1}\end{array}$ & $\mathrm{Mn}$ & \\
\hline T0 & 54,2 & $14,8 \mathrm{ab}$ & $155,8 \mathrm{a}$ & 52,2 & $62,4 \mathrm{~b}$ \\
T1 & 54,4 & $16,2 \mathrm{ab}$ & $138,2 \mathrm{ab}$ & 55,0 & $62,4 \mathrm{~b}$ \\
T2 & 52,2 & $14,4 \mathrm{ab}$ & $111,8 \mathrm{~b}$ & 50,4 & $61,4 \mathrm{~b}$ \\
T3 & 50,6 & $13,8 \mathrm{~b}$ & $138,8 \mathrm{ab}$ & 57,4 & $69,0 \mathrm{~b}$ \\
T4 & 47,2 & $13,4 \mathrm{~b}$ & $120,0 \mathrm{~b}$ & 56,0 & $75,2 \mathrm{~b}$ \\
T5 & 55,2 & $17,8 \mathrm{a}$ & $137,0 \mathrm{ab}$ & 65,2 & $96,0 \mathrm{a}$ \\
\hline F & 1,45 & 2,24 & 3,29 & 0,59 & 5,56 \\
Média & 52,3 & 15,1 & 133,6 & 56,0 & 71,1 \\
CV & 10,68 & 16,37 & 14,36 & 26,76 & 17,76 \\
\hline
\end{tabular}


Os teores foliares de B e Mn não variaram entre os tratamentos apresentados na Tabela 4 . Já o $\quad \mathrm{Cu} \quad$ o $\quad$ T5 apresentou superior ao $\mathrm{T} 3$ e ao $\mathrm{T} 4$, isto devido a maior quantidade de lodo aplicado. No caso do Fe o T0 apresentouse superior ao T2 e T4. Para o Zn o T5 apresentou superior a todos.
Com o aumento da dose de lodo compostado verifica-se na Figura 3 que no $\mathrm{Cu}$ e o Fe ocorreu um decréscimo do teor foliar no aumento da dose de lodo e posteriormente um acréscimo.
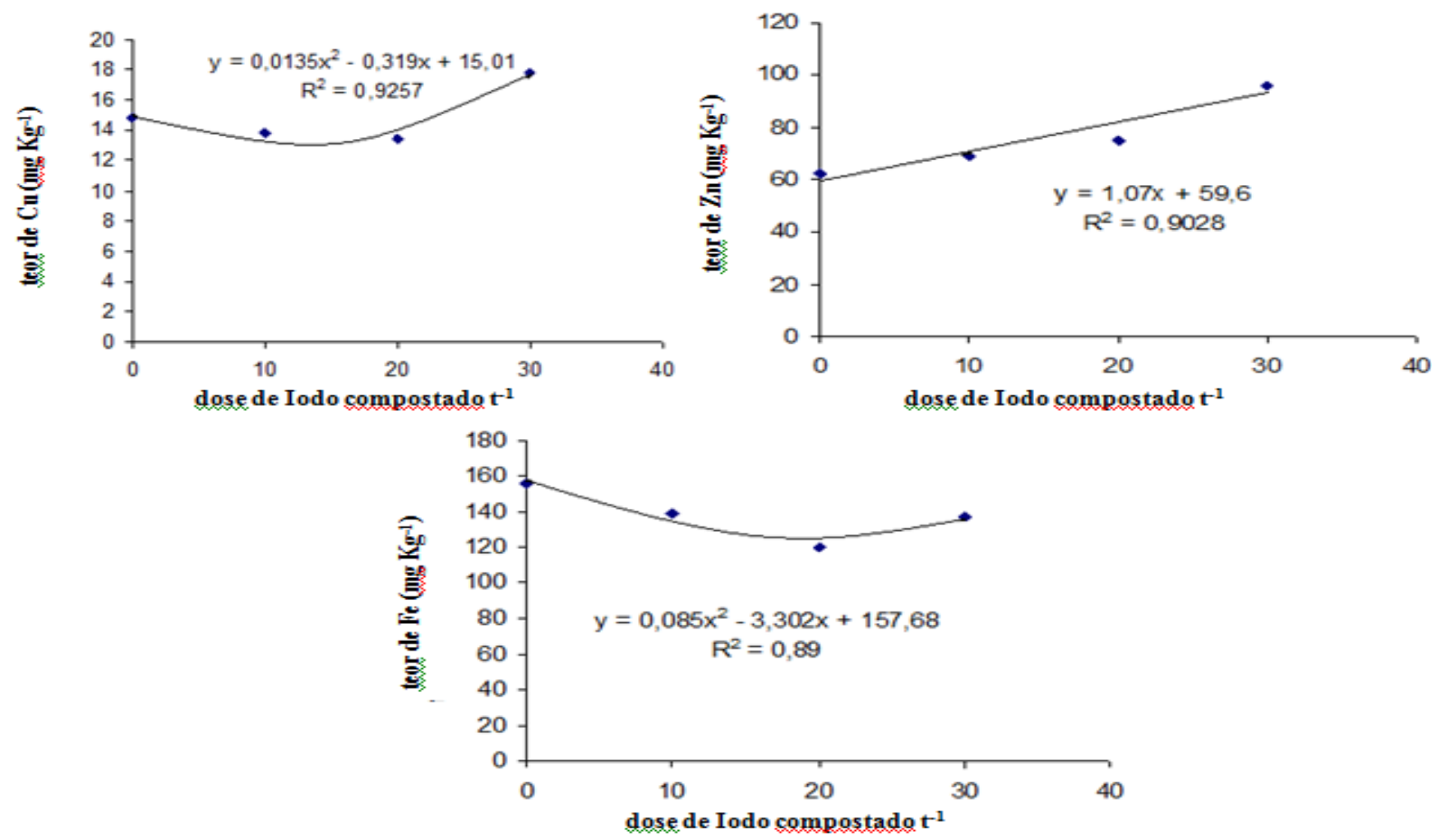

Figura 3 Teor foliar de micronutrientes no feijão em função da dose de lodo compostado

Para o Zn com aumento da dose de lodo ocorreu um acréscimo linear no teor foliar, substituindo os valores da equação verifica-se que se utilizássemos uma dosagem de 37 , $75 \mathrm{t} \mathrm{ha}^{-1}$ de lodo os teores foliares de $\mathrm{Zn}$ alcançaria o valor de $100 \mathrm{mg} \mathrm{kg}^{-1}$ que já seria um valor acima do adequado.

Teixeira et al. (2.008) observou que aumentando doses de $\mathrm{N}$ no feijão até $120 \mathrm{~kg} \mathrm{ha}^{-1}$ de $\mathrm{N}$ diminuiu o teor de Fe nas folhas, no qual pode ser observado neste ensaio que o T0 onde não foi $\mathrm{N}$ apresentou teor de Fe mais elevado que o T2, embora o lodo de esgoto apresente um teor de Fe elevado.

As produtividades do feijoeiro neste experimento foram os seguintes: T0 $-1.823 \mathrm{~kg} \mathrm{ha}^{-1}$; T1 $-2.297 \mathrm{~kg} \mathrm{ha}^{-1}$; T2
$-2.426 \mathrm{~kg} \mathrm{ha}^{-1} ; \mathrm{T} 3-2.537 \mathrm{~kg} \mathrm{ha}^{-1} ; \mathrm{T} 4-2.622 \mathrm{~kg} \mathrm{ha}^{-1}$ e T5 $2.865 \mathrm{~kg} \mathrm{ha}^{-1}$ e para cada tonelada de lodo compostado houve um incremento na produtividade de $25,63 \mathrm{~kg}$ de feijão (Lobo et al, 2012).

Verifica-se na Tabela 5 que no tratamento de $50 \%$ de $\mathrm{N}$ proveniente do lodo compostado combinado com $50 \% \mathrm{~N}$ mineral e o tratamento $(200 \% \mathrm{~N}$ lodo) apresentou uma exportação de $\mathrm{N}$ superior ao tratamento onde não foi aplicado N. A cada tonelada de lodo de esgoto houve um incremento de $0,63 \mathrm{~kg}$ de $\mathrm{N}$ exportado (Figura 4).

Tabela 5 Exportação de macronutrientes do feijão

\begin{tabular}{lllllll}
\hline Tratamento & $\mathrm{N}$ & $\mathrm{P}$ & $\mathrm{K}$ & $\begin{array}{c}\mathrm{Ca} \\
\mathrm{kg} \mathrm{ha}^{-1}\end{array}$ & $\mathrm{Mg}$ & $\mathrm{S}$ \\
\hline T0 & $49,62 \mathrm{~b}$ & $6,32 \mathrm{~b}$ & $21,87 \mathrm{~b}$ & $7,77 \mathrm{~b}$ & $4,74 \mathrm{~b}$ & 5,11 \\
T1 & $57,73 \mathrm{ab}$ & $6,34 \mathrm{~b}$ & $24,57 \mathrm{ab}$ & $12,18 \mathrm{ab}$ & $6,13 \mathrm{ab}$ & 3,97 \\
T2 & $69,84 \mathrm{a}$ & $8,07 \mathrm{a}$ & $29,12 \mathrm{ab}$ & $8,54 \mathrm{ab}$ & $5,98 \mathrm{ab}$ & 4,15 \\
T3 & $65,16 \mathrm{ab}$ & $7,81 \mathrm{ab}$ & $27,90 \mathrm{ab}$ & $10,89 \mathrm{ab}$ & $6,67 \mathrm{ab}$ & 4,53 \\
T4 & $64,91 \mathrm{ab}$ & $7,92 \mathrm{ab}$ & $28,83 \mathrm{ab}$ & $11,13 \mathrm{ab}$ & $6,81 \mathrm{ab}$ & 5,24 \\
T5 & $74,65 \mathrm{a}$ & $8,57 \mathrm{a}$ & $30,98 \mathrm{a}$ & $13,41 \mathrm{a}$ & $7,68 \mathrm{a}$ & 5,72 \\
\hline F & 2,68 & 3,34 & 2,17 & 1,87 & 2,21 & 0,65 \\
Média & 63,65 & 7,50 & 27,21 & 10,65 & 6,34 & 4,79 \\
CV & 17,06 & 13,78 & 16,74 & 29,37 & 20,93 & 35,16 \\
\hline
\end{tabular}

Médias seguidas pelas mesmas letras não diferem entre si pelo teste de Duncan 5\% de probabilidade. T0 - sem N, T1 -100\% AM, T2 - 50\% AM + 50\% LE, T4 - 150\% LE, T5 - 200\% LE, T3 - 100\% LE. AM, - Adubação mineral; LE - Lodo de esgoto 

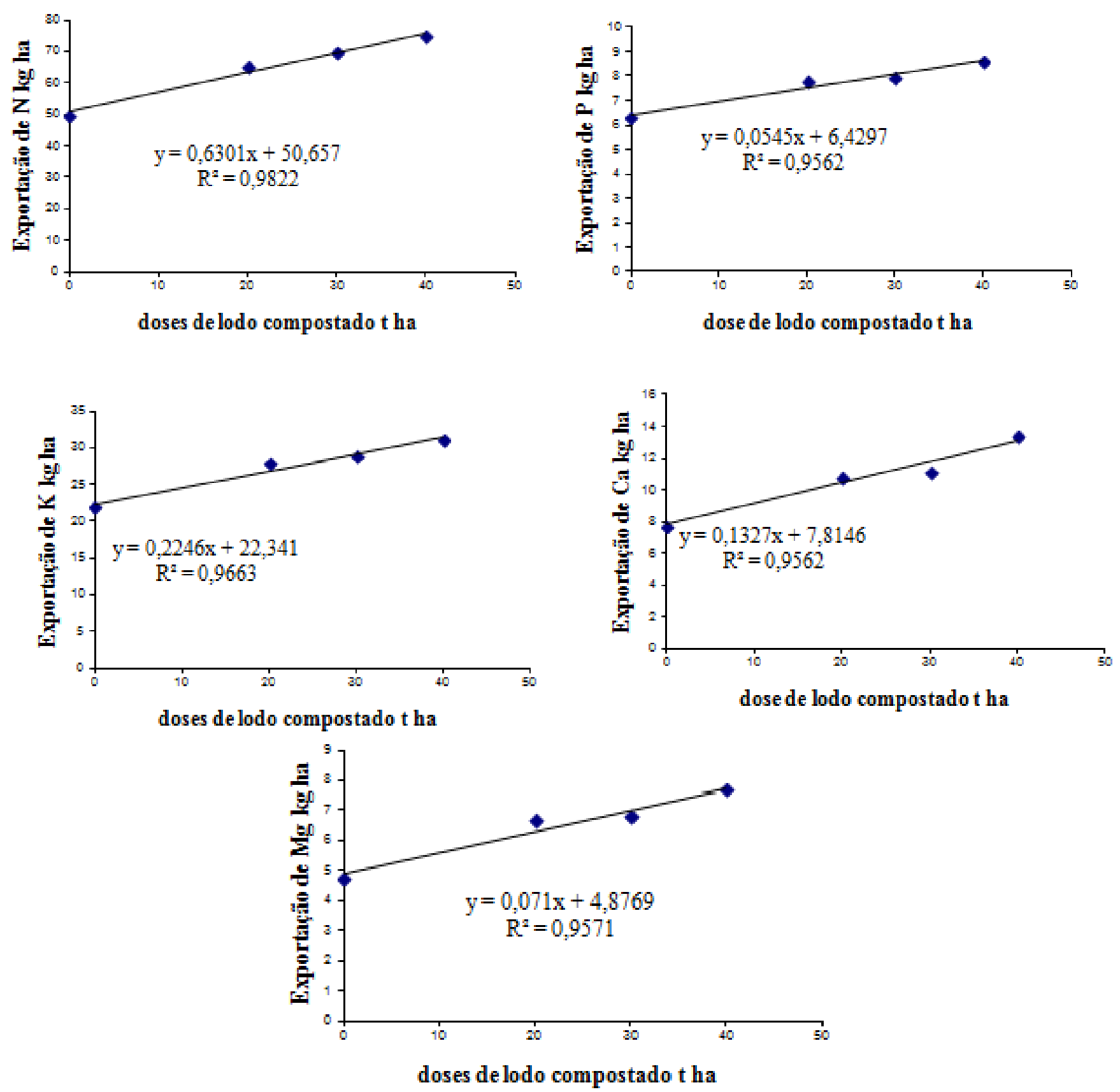

Figura 4 - Exportação de macronutrientes do feijão em função da dose de lodo compostado

Para o P apresentou-se uma exportação superior o tratamento (T2 e T5) em relação ao tratamento que não foi aplicado lodo (Tabela 5). Com o aumento da dose de lodo compostado aumentou a exportação de P linearmente (Figura 4). O lodo compostado apresenta uma elevada quantidade de $\mathrm{P}$ e este elemento é pouco perdido por lixiviação, sendo que este ensaio já esta com 4 aplicações de resíduo orgânico.

Quanto ao K o tratamento (200\% N lodo compostado), apresentou superior ao tratamento que não foi aplicado $\mathrm{N}$. Segundo Amaral et al. (1980), para a cultivar carioca a exportação de $\mathrm{K}$ esta entre 14 e $72 \mathrm{~kg} \mathrm{ha}^{-1}$, observa-se que todos os tratamentos estão nesta faixa. Com o aumento da dose de lodo compostado aumentou-se linearmente a exportação de K (Figura 4).
$\mathrm{Na}$ exportação de $\mathrm{Ca}$ e $\mathrm{Mg}$ verifica-se que o tratamento que recebeu uma maior dose de lodo (T5) exportou mais do que o tratamento que não recebeu $\mathrm{N}$ em função do aumento da produtividade. Houve um aumento linear crescente em função da dose de lodo compostado (Figura 4). Para o S não houve alteração na exportação de nutrientes entre os tratamentos.

Observa-se que a exportação de B foi superior no tratamento que receberam 100 e $200 \%$ do $\mathrm{N}$ proveniente do lodo em relação ao tratamento que não recebeu N (Tabela 6). Com o aumento da dose de lodo compostado ocorreu um aumento na exportação de uma forma quadrática (Figura 5). 
Tabela. 6. Exportação de micronutrientes do feijão

\begin{tabular}{llllll}
\hline Tratamento & $\mathrm{B}$ & $\mathrm{Cu}$ & $\mathrm{Fe}$ & $\mathrm{Mn}$ & $\mathrm{Zn}$ \\
& & & $\mathrm{g} \mathrm{ha}^{-1}$ & & \\
\hline T0 & $39,70 \mathrm{~b}$ & $13,24 \mathrm{~b}$ & $172,51 \mathrm{~b}$ & $20,53 \mathrm{~b}$ & $56,19 \mathrm{~d}$ \\
T1 & $57,78 \mathrm{ab}$ & $18,37 \mathrm{a}$ & $239,35 \mathrm{ab}$ & $28,98 \mathrm{a}$ & $67,31 \mathrm{~cd}$ \\
T2 & $48,83 \mathrm{ab}$ & $19,94 \mathrm{a}$ & $229,17 \mathrm{ab}$ & $30,16 \mathrm{a}$ & $72,99 \mathrm{bcd}$ \\
T3 & $64,21 \mathrm{a}$ & $22,60 \mathrm{a}$ & $243,79 \mathrm{ab}$ & $32,09 \mathrm{a}$ & $89,10 \mathrm{abc}$ \\
T4 & $56,32 \mathrm{ab}$ & $20,25 \mathrm{a}$ & $254,96 \mathrm{a}$ & $33,29 \mathrm{a}$ & $92,54 \mathrm{ab}$ \\
T5 & $63,35 \mathrm{a}$ & $22,00 \mathrm{a}$ & $288,66 \mathrm{a}$ & $32,44 \mathrm{a}$ & $103,17 \mathrm{a}$ \\
\hline F & 2,40 & 4,89 & 2,41 & 4,03 & 5,41 \\
Média & 55,03 & 19,40 & 238,07 & 29,58 & 80,22 \\
CV & 21,93 & 15,74 & 20,63 & 15,84 & 18,90 \\
\hline
\end{tabular}

Médias seguidas pelas mesmas letras não diferem entre si pelo teste de Duncan 5\% de probabilidade. T0 - sem N, T1 -100\% AM, T2 - 50\% AM + 50\% LE, T3 - 100\% LE, T4 - 150\% LE, T5 - 200\% LE AM - Adubação mineral; LE - Lodo de esgoto
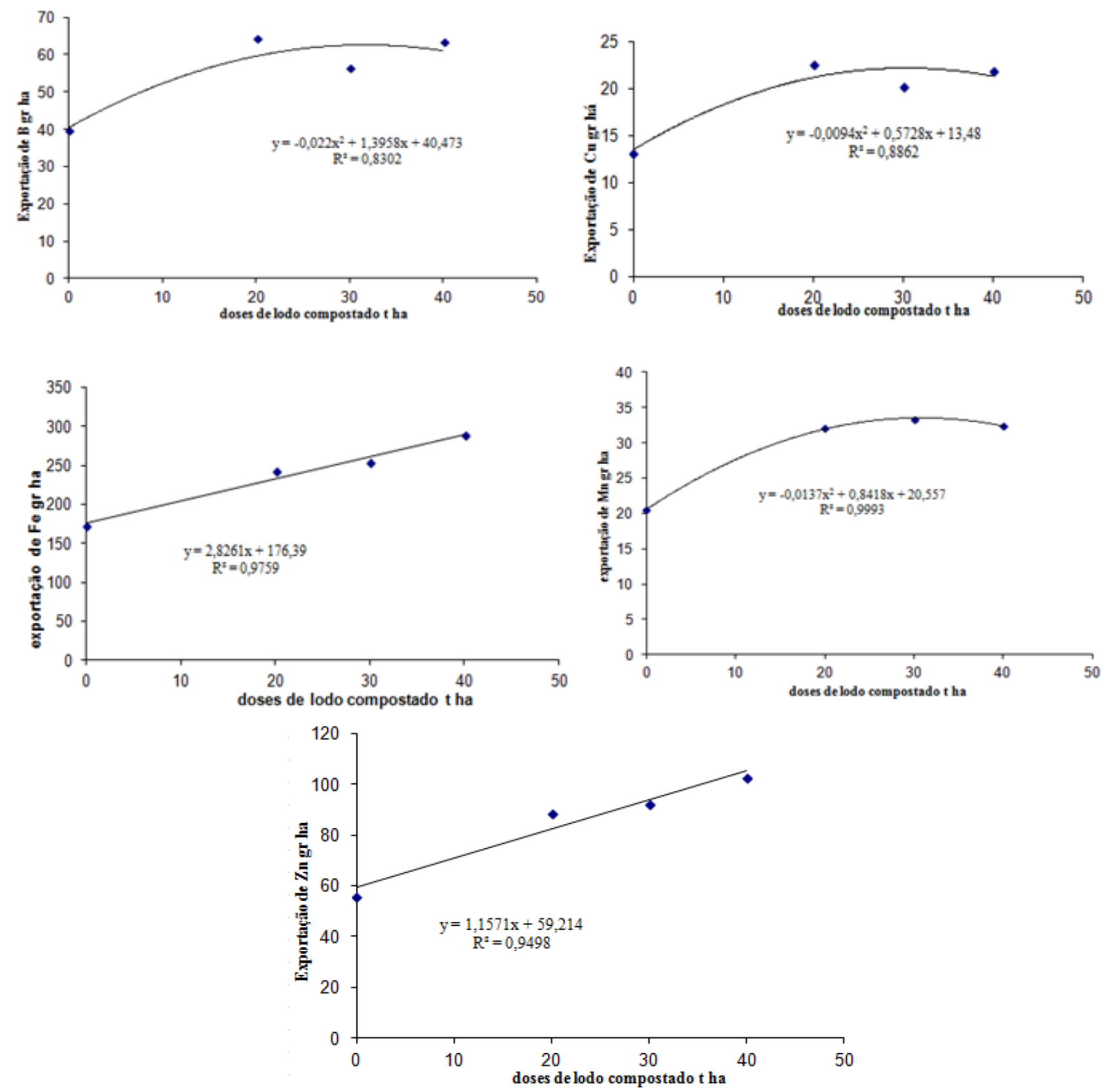

Figura 5 Exportaçào de micronutrientes do feijão em funçao da dose de lodo compostado

Para o $\mathrm{Cu}$ e $\mathrm{Mn}$ os tratamentos que receberam $\mathrm{N}$ exportaram mais em relação ao tratamento que não recebeu
N. Com aumento da dose de lodo compostado houve um incremento na exportação de uma forma quadrática. 
Quanto ao Fe observa-se na Tabela 6 que os tratamentos (T4 e T5) foram superiores em relação ao tratamento que não recebeu N. Com o aumento da dose de lodo compostado ocorreu um aumento na exportação de $\mathrm{Fe}$, conforme apresenta a Figura 5.

$\mathrm{O} \mathrm{Zn}$ foi o micronutriente que mais variou na exportação, quanto maior foi a dosagem de lodo de esgoto maior foi a sua exportação, os tratamentos onde receberam $\mathrm{N}$ mineral e não receberam $\mathrm{N}$ não variaram a exportação de $\mathrm{Zn}$.

\section{CONCLUSÕES}

$\mathrm{O}$ aumento da dose de lodo influenciou nops teores foliares de $\mathrm{Ca}, \mathrm{P}, \mathrm{Mg}, \mathrm{Cu}, \mathrm{Fe}$ e $\mathrm{Zn}$.

A exportação de $\mathrm{N}, \mathrm{P}, \mathrm{K}, \mathrm{Ca}, \mathrm{Mg}, \mathrm{B}, \mathrm{Cu}, \mathrm{Fe}, \mathrm{Mn}$ e $\mathrm{Zn}$ influenciaram com o aumento da dose de lodo.

$\mathrm{O} N$ não interferiu nos teores foliares.

$\mathrm{O} \mathrm{N}$ proporcionou uma maior exportação de $\mathrm{Cu}$ e $\mathrm{Mn}$.

\section{REFERÊNCIAS BIBLIOGRÁFICAS}

AMARAL SOBRINHO, N.M.B.; VELLOSO, A.C.X.; OLIVEIRA, C. Solubilidade de metais pesados em solos tratados com resíduos siderúrgico. Revista Brasileira de Ciência do Solo, Viçosa, v. 21, n. 1, p. 9-16, 1997.

ARF, O. et al. Mecanismos de abertura do sulco e adubação nitrogenada no cultivo do feijoeiro em sistema plantio direto. Bragantia, Campinas, v.67, n.2, p. 499-506, 2008. (p)

BARBOSA, G. M. C; FILHO, J. T. Uso agrícola do lodo de esgoto: influência nas propriedades químicas e físicas do solo, produtividade e recuperação de áreas. Revista Semina: Ciências Agrárias, Londrina, v. 27, n. 4, p. 565-580, out./dez. 2006.

BARBOSA, G. M. et al. Efeito residual do lodo de esgoto na produtividade do milho safrinha. Revista Brasileira de Ciência do Solo, Londrina, p. 601-605, 2007.

BETIOL, W.; CAMARGO, O.A. Lodo de esgoto na agricultura: potencial de uso e problemas. TEC Hoje, Belo Horizonte, p. 1-4, 2007.

BINOTTI, F.F. da S. et al. S. Manejo do solo e da adubação nitrogenada na cultura do feijão de inverno e irrigado. Bragantia, Campinas, v. 66, n.1, p.121-129, 2007.

CUNNINCHAM, J. D.; KEENEY, D. R.; RYAN, J. A. Yeald and metal composition of crop and rye grown on sewage amended soil. Journal Environmrnt Quality, Madilson, v. 4, p.448-454, 1975.

COMPANIA NACIONAL DO MEIO AMBIENTE. Resolução no 375/2006, 2006. Apresenta resoluções do CONAMA. Disponível

<htb://www.mma.gov.br/post/conama

/legiano/>. Acesso em: 10 de out de 2006.

CORRÊA, J.C.; BÜLL, L.T.; CRUSCIOL, C.A.C.; MARCELINO, R. \& MAUAD, M. Correção da acidez e mobilidade de íons em Latossolo com aplicação superficial de escória, lamacal, lodos de esgoto e calcário. Pesquisa Agropecuária Brasileira, Brasília, v.42, n.9, p.1307-1317, set. 2007.
CORRÊA, J.C et al. Aplicação superficial de diferentes fontes de corretivos no crescimento radicular e produtividade da aveia preta. Revista Brasileira de Ciência do Solo, Brasília, p. 1583-1590, 2008.

CAMPANHARO, M. Acidez do solo na fixação biológica de nitrogênio em feijoeiro comum. . Revista Brasileira de Ciências Agrárias, Recife, v.5, n.3, p.285-290, 2010.

EMPRESA BRASILEIRA DE PESQUISA AGROPECUARIA. Serviço de produção de informação (Brasília - DF). Recomendações técnicas para o cultivo do feijão: zonas 61 e 83. Brasília, 1993. 93p.

JOSE, E. M. Níveis de nitrogênio em cobertura no desenvolvimento vegetativo e na severidade de Pseudomonas syringaePv. Tabaci em feijoeiro IACcarioca. Botucatu. 2001. 53f. Dissertação (mestrado) Faculdade de Ciencias Agronomicas, Universidade Estadual Paulista, Botucatu, 2001.

KITAMURA, A. E. et al. Recuperação de um solo degradado com aplicação de adubos verdes e lodo de esgoto. Revista Brasileira de Ciência do Solo, p 405-416, 2008.

LANARV. Análise de corretivos, fertilizantes e inoculantes: métodos oficiais. Brasília: Ministério da Agricultura, 1988. 104p.

LOBO, T. F. et al. Efeito do nitrogênio e do lodo de esgoto nos fatores produtivos do feijoeiro. Revista Ceres, Viçosa, v.59, n.1, p. 601-605, 2012.

MALAVOLTA, E., VITTI G. C., OLIVEIRA S. A. Avaliação do estado nutricional das plantas: Princípios, métodos e técnicas de avaliação do estado nutricional. $2^{\mathrm{a}}$ edição. Piracicaba - SP. Editora Potafos, 1997. 319p.

OLIVEIRA, I. P. de; ARAUJO, R. S.; DUTRA, L. G. Nutrição mineral e fixação biológica de nitrogênio. In: ARAUJO, R.S. et al. (eds). Cultura do feijão comum no Brasil. Piracicaba, Associação Brasileira para pesquisa de potassa e do fosfato, p. 169-221, 1996.

PIMENTEL GOMES, F. Curso de estatística experimental. 14. ed. Piracicaba: ed. Do Autor, 2000. 477p.

TEIXEIRA, C.M. et al. Produtividade e teores foliares de nutrientes do feijoeiro sob diferentes palhadas e doses de nitrogênio em semeadura direta ura direta. Acta Scientiarum. Agronomy, Maringá, v. 30, n. 1, p. 123130, 2008.

VALDERRAMA, M. et al. Fontes e doses de nitrogênio e fósforo em feijoeiro no sistema plantio direto. Pesquisa Agropecuária Tropical, Goiánia, v.39, n.3, p. 191-196, 2009.

WISNIEWSKI, C. et al. Uso do lodo de esgoto da ETE Belém na recuperação de áreas degradadas por mineração de calcário. SANARE, Curitiba, v.5, p. 7685, 1996. 